\title{
The Dispersion of Gravitational Waves
}

\author{
J. MADORE \\ Physique Théorique, Institut Henri Poincaré, 11 Rue Pierre et Marie Curie, Paris, France
}

Received March 14; in revised form April 19, 1972

\begin{abstract}
A definition is given of a plane gravitational wave in a curved background space-time manifold. For a particular background metric, a dispersion relation for the waves is derived analogous to that satisfied by plane electromagnetic waves in a dilute plasma.
\end{abstract}

\section{Introduction}

Our purpose here is to discuss the dispersion of gravitational waves in vacuo. We give a definition of a plane wave in a curved background space-time manifold and for a particular background metric a dispersion relation for these waves is derived. We formulate the results in a general manner but because of certain ad hoc assumptions we are forced to make, we can offer no reason to believe that they are valid for any background metric other than that given as an example.

In comparison, we first give the electromagnetic case in Section II. This is a test of the validity of the wave ansatz. There can be no dispersion of electromagnetic radiation in the absence of charged matter.

\section{II}

In this section we shall discuss electromagnetic waves in a region of space-time in which there is no charged matter present. If there is neutral matter then we suppose that the dielectric constant and the permeability are both equal to one.

An electromagnetic wave is described by a Maxwell tensor of the form

$$
\tilde{F}_{\mu v}=F_{\mu v}+\varepsilon A_{\mu v},
$$

where $F_{\mu \nu}\left(x^{\sigma}\right)$ describes an arbitrary given background electromagnetic field. $\varepsilon$ is a dimensionless constant characterizing the order of magnitude of the amplitude of the wave and $A_{\mu \nu}$ is given by

where

$$
A_{\mu v}=\partial_{\mu} A_{v}-\hat{\partial}_{v} A_{\mu},
$$

$$
A_{\mu}=A_{\mu}\left(x^{\sigma}, \omega \phi\right) \text {, }
$$


the potential of the wave, is a function explicitly of the point in space-time $x^{\sigma}$ as well as of a constant $\omega$ and a phase $\phi . \phi$ is a function of $x^{\sigma} . \omega$ has the dimension of (length) $)^{-1}$ and $\phi$ has the dimension of length. For the moment $\phi$ is arbitrary. We shall see that once an explicit functional dependence of $A_{\mu}$ on $\omega \phi$ is given then Maxwell's equations will place restrictions on $\phi$.

Define the normal to the family of hypersurfaces $\phi=$ const:

$$
\xi_{\sigma}=\partial_{\sigma} \phi \text {. }
$$

$\xi_{\sigma}$ is a dimensionless vector field. The frequency of the wave as observed by an observer $u^{\alpha}$ with $u^{2}=1$ is $\omega u \cdot \xi$.

This factorization of the observed frequency $\omega u \cdot \xi$ into $\omega$ and $u \cdot \xi$ is rather arbitrary. We can for example choose $c \phi$ as phase where $c$ is an arbitrary constant. Then the observed frequency would be factorized as $(\omega / c)(u \cdot \xi c)$. The problem is that frequency is an observer dependent quantity.

In most situations of physical interest there will be a coordinate system in which the explicit calculations will be carried out, for example if the background metric is stationary. We shall fix $\omega$ sufficiently for our purposes by demanding that when the observer is at rest in this coordinate system, then $u \cdot \xi$ is of the order of magnitude of one. This means then that $\omega$ is of the order of magnitude of the frequency in a particular adapted coordinate system.

Define the parameter $r$ by the equations

Set

$$
\frac{d x^{\alpha}}{d r}=\xi^{\alpha}
$$

Then

$$
\dot{A}_{\mu}=\frac{\partial A_{\mu}}{\partial(\omega \phi)}, \quad A_{\mu_{.} \lambda}=\left(\partial_{\lambda} A_{\mu}\right)_{\phi=\mathrm{const}} .
$$

We have therefore

$$
\partial_{\lambda} A_{\mu}=\omega \xi_{\lambda} \dot{A}_{\mu}+A_{\mu, \lambda} \text {. }
$$

$$
A_{\mu \nu}=\omega \dot{A}_{[v} \xi_{\mu]}+A_{[v, \mu]} .
$$

The background electromagnetic field satisfies Maxwell's equations:

$$
D^{v} F_{\mu v}=0 .
$$

We assume that $\varepsilon$ is small enough that the electromagnetic wave does not ionize the matter. We have then that the perturbed electromagnetic field $\tilde{F}_{\mu l}$ satisfies also Maxwell's equations:

Set

$$
D^{v} \tilde{F}_{\mu \nu}=0 \text {. }
$$

$$
A_{\lambda: \mu}=\left(D_{\mu} A_{\lambda}\right)_{\phi=\text { const }} \text {. }
$$


We have from (2.5) and (2.6) the following equations for the potential of the wave:

$$
\omega^{2} \ddot{A}_{[v} \xi_{\mu]} \xi^{v}+\omega\left(\xi_{[\mu} \dot{A}_{v]}{ }^{v}+\dot{A}_{[v: \mu]} \xi^{\prime \prime}+\dot{A}_{[v} \xi_{\mu]}{ }^{\prime \prime}\right)+A_{[v ; \mu] ;}{ }^{\prime}=0 .
$$

These equations may be simplified by imposing a gauge condition. If we demand that the Lorentz gauge condition hold,

then Eq. (2.7) becomes

$$
D_{1} A^{v}=0 \text {, }
$$

$$
\omega^{2} \xi^{2} \ddot{A}_{\mu}+\omega\left(2 \frac{\delta \dot{A}_{\mu}}{\delta r}+\dot{A}_{\mu} \xi_{; \lambda}^{\lambda}\right)+A_{\mu ; ; \lambda}^{\lambda}+R_{\mu}, A^{\lambda}=0 .
$$

$R_{\mu \nu}$ is the Ricci tensor of the background metric. $\delta / \delta r \equiv_{; \alpha} \xi^{\alpha}$. We now assume an explicit functional dependence of $A_{\mu}\left(x^{\sigma}, \omega \phi\right)$ on $\omega \phi$. We assume that in the Lorentz gauge $(2.8) A_{\mu}\left(x^{\sigma}, \omega \phi\right)$ is given by

$$
\left.A_{\mu}\left(x^{\sigma}, \omega\right) \phi\right)=a_{\mu}\left(x^{\sigma}\right) e^{i \omega \phi},
$$

where $a_{\mu}\left(x^{\sigma}\right)$ is a real vector. Eqs. (2.8) and (2.9) then yield the following equations for $a_{\mu}$ :

$$
\begin{gathered}
\left(\xi_{\mu} a^{\mu}-\frac{i}{\omega} a_{; \mu}^{\mu}\right) e^{i(\omega)}=0 . \\
{\left[\omega^{2} \xi^{2} a_{\mu}-\left(a_{\mu ; ; \lambda}^{\lambda}+R_{\mu \lambda} a^{\lambda}\right)-i \omega\left(2 \frac{\delta a_{\mu}}{\delta r}+a_{\mu} \xi_{; \lambda}^{\lambda}\right)\right] e^{i \omega \phi}=a .}
\end{gathered}
$$

The assumption (2.10) is very strong. The system of Eqs. (2.8), (2.9) has always a solution for arbitrary phase $\phi$ and background metric. The existence of a solution to the system (2.11), (2.12) imposes very severe restrictions both on the phase and on the background metric.

In addition to (2.10) we shall assume that the Eqs. (2.11) and (2.12) are valid as complex equations. This means that the change in $a_{\mu}\left(x^{\sigma}\right)$ and in the background metric $g_{\mu v}\left(x^{\sigma}\right)$ can be neglected when the phase $\phi$ changes by $\pi / 2 \omega$. Let $L^{\prime}$ and $L^{\prime \prime}$ be the characteristic lengths in which the change in respectively $a_{\mu}$ and $g_{\mu v}$ becomes significant. Then the above assumption will be valid if the following inequality is satisfied:

$$
\omega \gg 1 / L \equiv \max \left(1 / L^{\prime}, 1 / L^{\prime \prime}\right) .
$$

In the important special case where the metric is stationary, the phase is of the form

$$
\phi \equiv x^{0}-f\left(x^{i}\right) .
$$


If $a_{\mu}$ does not depend on the preferred time coordinate $x^{0}$ then the change in $a_{\mu}$ and $g_{\mu \nu}$ when the phase $\phi$ changes by $\pi / 2 \omega$ can be neglected for all values of $\omega$.

Setting the real and imaginary parts of Eqs. (2.11), (2.12) separately equal to zero yields the following four equations:

$$
\begin{aligned}
a^{\mu} \xi_{\mu} & =0, \\
a_{; \mu}^{\mu} & =0, \\
\xi^{2} a_{\mu}-\frac{1}{\omega^{2}}\left(a_{\mu: ; \lambda}{ }^{\lambda}+R_{\mu \lambda} a^{\lambda}\right) & =0, \\
2 \frac{\delta a_{\mu}}{\delta r}+\xi_{; \lambda}^{\lambda} a_{\mu} & =0 .
\end{aligned}
$$

An electromagnetic field described by a potential of the form (2.10) where $a_{\mu}$ is a solution to these four equations in the absence of sources, will be called a wave. If also

$$
\left|a_{\mu ; ; \lambda}{ }^{\lambda}+R_{\mu \lambda} a^{\lambda}\right| \ll \omega^{2}\left|a_{\mu}\right|,
$$

so that the second term on the left-hand side of Eq. $(2.14 \mathrm{c})$ can be put equal to zero, then we shall say that the wave is a plane wave. One sees that (2.15) is an immediate consequence of (2.13).

Before giving two examples of plane waves, we draw some immediate consequences from Eqs. (2.14), (2.15) [1,2]. From (2.14c), (2.15) we see that the hypersurfaces of constant phase are null and the integral curves of the vector field $\xi^{\mu}$ are null geodesics. If we set

$$
a_{\mu}=a n_{\mu}, \quad n^{2}=-1,
$$

then from Eq. (2.14d) we obtain the conservation equation

$$
\left(a^{2} \xi^{\mu}\right)_{; \mu}=0,
$$

and the transport equation

$$
\frac{\delta n_{\mu}}{\delta r}=0 .
$$

The simplest example of a plane wave is the classical example in flat space. Let $a_{\mu}$ be an arbitrary space-like parallel vector field satisfying Eq. (2.14a) with $\xi_{\mu}$ an arbitrary null parallel vector field. Eq. (2.15) is satisfied for all $\omega$.

An example of a plane wave can also be given in a space-time manifold which is not flat. Consider $\mathbb{R}^{4}$ with the coordinate system

$$
\left(x^{\mu}\right)=\left(t, y, x^{2}, x^{3}\right) .
$$


In the region $y>0$ introduce the metric [6] whose components are

$$
g_{\mu v}=\operatorname{diag}\left(e^{2 v},-1,-e^{2 \lambda},-e^{2 \lambda}\right),
$$

where $\lambda, \mu$ are functions of $y$ alone. The two-dimensional surfaces $t=$ const, $y=$ const are homogeneous and isotropic. The total isometry group is the product of the group of motions of the euclidian plane by the real line.

The vacuum field equations are

$$
\begin{aligned}
2 \lambda^{\prime \prime}+3\left(\lambda^{\prime}\right)^{2} & =0, \quad \lambda^{\prime} \equiv \frac{\partial \lambda}{\partial y}, \\
2 v^{\prime}+\lambda^{\prime} & =0 .
\end{aligned}
$$

When these equations are satisfied, the non-vanishing components of the Riemann tensor are

$$
\begin{gathered}
R_{101}^{0}=-\left(\hat{\lambda}^{\prime}\right)^{2}, \quad R_{323}^{2}=-\left(\lambda^{\prime}\right)^{2} e^{2 \lambda} \\
R_{202}^{0}=R_{303}^{0}=R_{212}^{1}=R_{313}^{1}=\frac{\left(\lambda^{\prime}\right)^{2}}{2} e^{2 \lambda} .
\end{gathered}
$$

Let $a_{2}^{0}, a_{3}^{0}$ be two arbitrary constants. On one of the hypersurfaces $y=$ const introduce a vector field $a_{\mu}^{0}$ whose components in the system of coordinates (2.18) are

$$
a_{\mu}^{0}=\left(0,0, a_{2}^{0}, a_{3}^{0}\right) .
$$

Introduce a phase $\phi$ given by

$$
\phi \equiv t-f(y) .
$$

Extend $a_{\mu}^{0}$ to a vector field $a_{\mu}$ in the region $y>0$ using Eq. (2.14d). One can show that for any $\omega$, the remaining Eq. (2.14) and Eq. (2.15) are then satisfied if one chooses for $f$ a solution to the equation

$$
\xi^{2}=0 \text {. }
$$

The electromagnetic waves are therefore not dispersed by the background metric.

\section{III}

The calculations of the preceeding section may be formally carried over to the case of gravitational waves in a straightforward manner. However we shall see that it is not clear how one should formulate the plane wave condition analogous to (2.15). Using as example the metric introduced in Section II we can eliminate this ambiguity, but this is 
done in such an ad hoc manner that we can give no reason for believing that the dispersion relation we find is valid for any other background metric.

A gravitational wave is described by a metric tensor with components of the form

$$
\tilde{g}_{\mu v}=g_{\mu v}+\varepsilon h_{\mu v},
$$

where $g_{\mu v}$ are the components of an arbitrary given background metric. $\varepsilon$ is a dimensionless parameter characterizing the order of magnitude of the wave and

$$
h_{\mu v}=h_{\mu v}\left(x^{\sigma}, \omega \phi\right),
$$

the potential of the wave is a function explicitly of the point in space-time $x^{\sigma}$ as well as of a constant $\omega$ and a phase $\phi . \omega$ and $\phi$ as well as $r, \xi_{\mu}$ and the observed frequency are defined as in Section II.

Set

$$
\dot{h}_{\mu v}=\frac{\partial h_{\mu v}}{\partial(\omega \phi)}, \quad h_{\mu v, \lambda}=\left(\partial_{\lambda} h_{\mu v}\right)_{\phi=\text { const }} .
$$

Then

$$
\partial_{j} h_{\mu v}=\omega \xi_{\lambda} \dot{h}_{\mu v}+h_{\mu v, \lambda} .
$$

The most general coordinate transformation of the background space-time manifold is of the form

$$
x^{\prime \mu}=F^{\mu}\left(x^{\alpha}\right) .
$$

To this we shall add a gauge transformation of the potential of the wave, so that the most general admissible transformation of the components of the perturbed metric is given by

$$
\tilde{g}_{\mu v}=\tilde{g}_{\varrho \sigma}^{\prime} \frac{\partial x^{\prime} \underline{\partial}}{\partial x^{\mu}} \frac{\partial x^{\prime \sigma}}{\partial x^{\nu}},
$$

where $x^{\prime \mu}\left(x^{x}\right)$ is of the form

$$
x^{\prime \mu}=F^{\prime \mu}\left(x^{\alpha}\right)+\frac{\varepsilon}{\omega} f^{\prime \mu}\left(x^{\alpha}, \omega \phi\right) .
$$

Define

$$
f_{\mu}=\frac{\partial F^{\prime \lambda}}{\partial x^{\mu}} g_{\lambda \sigma}^{\prime} f^{\prime \sigma}
$$

where $g_{\lambda \sigma}^{\prime}$ are the components of the background metric in the coordinate system $F^{\prime \mu}\left(x^{\alpha}\right)$. Define

$$
h_{\mu v: \lambda}=\left(D_{\lambda} h_{\mu v}\right)_{\phi=\text { const }} .
$$


Then one readily sees that the potential of the wave in the coordinate system $F^{\prime \mu}\left(x^{\alpha}\right)$ is $h_{\mu \nu}^{\prime}$ given by

$$
h_{\mu v}=h_{\varrho \sigma}^{\prime} \frac{\partial F^{\prime} \varrho}{\partial x^{\mu}} \frac{\partial F^{\prime \sigma}}{\partial x^{v}}+\dot{f}_{(\mu} \xi_{v)}+\frac{1}{\omega} f_{(\mu ; v)} .
$$

Here and in what follows we have neglected powers of $\varepsilon$ greater than one. We shall discuss shortly what this means physically.

The components of the affine connection in the perturbed and unperturbed metric are related by the equation

$$
\tilde{\Gamma}_{\mu v}^{\lambda}=\Gamma_{\mu v}^{\lambda}+\frac{\varepsilon \omega}{2}\left(\xi_{(\mu} \dot{h}_{v)}^{\lambda}-\xi^{\lambda} \dot{h}_{\mu v}\right)+\frac{\varepsilon}{2}\left(h_{(\mu ; v)}^{\lambda}-h_{\mu v ;}{ }^{\lambda}\right) .
$$

The components of the Riemann tensor are related as follows:

$$
\tilde{R}_{\mu \sigma \nu}^{\lambda}=R_{\mu \sigma \nu}^{\lambda}+\frac{\varepsilon \omega^{2}}{2} A_{\mu \sigma \nu}^{(2) \lambda}+\frac{\varepsilon \omega}{2} A_{\mu \sigma \nu}^{(1) \lambda}+\frac{\varepsilon}{2} A_{\mu \sigma \nu}^{(0) \lambda},
$$

where the quantities $A_{\mu \sigma v}^{(i) ;}$ are given by

$$
\begin{aligned}
& A_{\lambda \mu \sigma v}^{(2)}=\xi_{[\lambda} \ddot{h}_{\mu][\sigma} \xi_{v]}, \\
& A_{\lambda \mu \sigma v}^{(1)}=\xi_{[\sigma} \dot{h}_{\mathrm{v}][\lambda ; \mu]}+\xi_{[\lambda} \dot{h}_{\mu][\sigma ; v]}-\xi_{\lambda ;[\sigma} \dot{h}_{v] \mu}+\xi_{\mu ;[\sigma} \dot{h}_{v] \lambda}, \\
& A_{\lambda \mu \sigma v}^{(0)}=h_{v[\lambda ; \mu] ; \sigma}-h_{\sigma[\lambda ; \mu] v}+h_{\lambda \mu ;[v ; \sigma]} .
\end{aligned}
$$

The components of the Ricci tensor are related as follows:

$$
\tilde{R}_{\mu \sigma}=R_{\mu \sigma}+\frac{\varepsilon \omega^{2}}{2} A_{\mu \sigma}^{(2)}+\frac{\varepsilon \omega}{2} A_{\mu \sigma}^{(1)}+\frac{\varepsilon}{2} A_{\mu \sigma}^{(0)},
$$

where the quantities $A_{\mu \sigma}^{(i)}$ are given by

$$
\begin{aligned}
& A_{\mu \sigma}^{(2)}=\xi^{\lambda} \ddot{h}_{\mu[\sigma} \xi_{\lambda]}-\xi_{\mu} \ddot{h}_{[\sigma}^{\lambda} \xi_{\lambda]}, \\
& A_{\mu \sigma}^{(1)}=\xi_{[\sigma} \dot{h}_{\lambda] ; \mu}^{\lambda}-\xi_{[\sigma} \dot{h}_{\lambda] \mu ;}{ }^{\lambda}+\xi^{\lambda} \dot{h}_{\mu[\sigma ; \lambda]} \\
& -\xi_{\mu} \dot{h}_{[\sigma ; \lambda]}^{\lambda}-\xi_{;[\sigma}^{\lambda} \dot{h}_{\lambda] \mu}+\xi_{\mu ;[\sigma} \dot{h}_{\lambda]}^{\lambda}, \\
& A_{\mu \sigma}^{(0)}=h_{\lambda ; \mu ; \sigma}^{\lambda}-h_{(\sigma ; \mu) ; \lambda}^{\lambda}+h_{\mu \sigma ; ; \lambda}{ }^{\lambda} \text {. }
\end{aligned}
$$

The expressions for $A_{\mu \sigma}^{(i)}$ may be simplified by imposing a gauge condition on the potential of the wave. If we demand that the deDonder condition hold:

$$
D^{\lambda}\left(h_{\lambda \mu}-\frac{h_{\sigma}^{\sigma}}{2} g_{\lambda \mu}\right)=0
$$


then the expressions for $A_{\mu \sigma}^{(i)}$ become

$$
\begin{aligned}
& A_{\mu \sigma}^{(2)}=\xi^{2} \ddot{h}_{\mu \sigma}, \\
& A_{\mu \sigma}^{(1)}=2 \frac{\delta \dot{h}_{\mu \sigma}}{\delta r}+\xi_{; \lambda}^{\lambda} \dot{h}_{\mu \sigma}, \\
& A_{\mu \sigma}^{(0)}=h_{\mu \sigma ; i \lambda}+h_{(\sigma}^{o} R_{\mu) \varrho}-2 R_{\alpha \mu \sigma \beta} h^{\alpha \beta} .
\end{aligned}
$$

The condition (3.11) may always be imposed by a transformation of the form (3.4) with $F^{\mu}\left(x^{x}\right) \equiv x^{\mu}$.

We shall suppose that there is no matter present. The background metric and the perturbed metric both satisfy the vacuum field equations:

$$
\tilde{R}_{\mu \nu}=0, \quad R_{\mu v}=0 .
$$

We have therefore the following equation for the potential of the wave:

$$
\omega^{2} A_{\mu \nu}^{(2)}+\omega A_{\mu \nu}^{(1)}+A_{\mu \nu}^{(0)}=0 \text {. }
$$

Before proceeding let us consider the relation of $\varepsilon$ to $\omega$. Let $L$ be the characteristic length in which the change in the components of the background metric becomes significant:

$$
\left|g_{\alpha \beta, \gamma}\right| \sim L^{-1}\left|g_{\alpha \beta}\right| .
$$

Since $h_{\alpha \beta}$ satisfies Eq. (3.14), $L$ is also the characteristic length for the amplitude of the potential.

Suppose that $L^{-1}$ is less than $\omega$. The left-hand side of Eq. (3.14) is the sum of three terms of which the smallest is of the order of $L^{-2}$. The largest term which we have neglected by not including terms quadratic in $\varepsilon$ is of the order $s \omega \omega^{2}$. We have therefore supposed that

$$
\varepsilon \ll 1 / L^{2} \omega^{2} \text {. }
$$

This means in particular that we have supposed that the energy of the wave $\left(\sim \varepsilon^{2} \omega^{2}\right)$ is negligeable compared with the energy of the background field $\left(\sim L^{-2}\right)$.

We now assume an explicit functional dependence of $h_{\mu v}\left(x^{\alpha}, \omega \phi\right)$ on $\omega \phi$. We assume that in the deDonder gauge (3.11), $h_{\mu v}\left(x^{\alpha}, \omega \phi\right)$ is given by

$$
h_{\mu v}\left(x^{\alpha}, \omega \phi\right)=a_{\mu v}\left(x^{\alpha}\right) e^{i \omega \phi},
$$

where $a_{\mu v}\left(x^{\alpha}\right)$ is a real tensor. One sees from the gauge transformation (3.5) that this ansatz is not gauge invariant. If $a_{\mu v}$ is real in one gauge, it will be in general complex in another gauge. 
Eqs. (3.11) and (3.14) yield the following equations for $a_{\mu \nu}$ :

$$
\begin{array}{r}
\left.\left[\left(a_{\mu}^{v}-\frac{a}{2} \delta_{\mu}^{v}\right) \xi_{v}-\frac{i}{\omega}\left(a_{\mu}^{v}-\frac{a}{2} \delta_{\mu}^{v}\right)\right]_{: v}\right] e^{i \omega \phi}=0, \\
{\left[\omega^{2} \xi^{2} a_{\mu \nu}-\left(a_{\mu \nu ; ; \lambda}^{\lambda}-2 R_{\varrho \mu \nu \sigma} a^{\varrho \sigma}\right)-i \omega\left(2 \frac{\delta a_{\mu \nu}}{\delta r}+\xi_{; \lambda}^{\lambda} a_{\mu \nu}\right)\right] e^{i \omega \phi}=0 .}
\end{array}
$$

We have set $a_{\lambda}^{\lambda}=a$.

The assumption (3.16) is very strong. The system of Eqs. (3.11), (3.14) has always a solution for arbitrary phase $\phi$ and background metric. The existence of a solution to the system (3.17), (3.18) imposes very severe restrictions both on the phase and on the background metric.

In addition to (3.16) we shall assume that (3.17) and (3.18) are valid as complex equations. The conditions for this are the same as in Section II. Setting the real and imaginary parts of Eqs. (3.17), (3.18) separately equal to zero yields the following four equations:

$$
\begin{aligned}
a_{\mu}^{v} \xi_{v} & =\frac{a}{2} \xi_{\mu}, \\
a_{\mu ; v}^{v} & =\frac{a_{\mu}}{2}, \\
\xi^{2} a_{\mu v} & =\frac{1}{\omega^{2}}\left(a_{\mu v ; ; \lambda}^{\lambda}-2 R_{\varrho \mu v \sigma} a^{\varrho \sigma}\right), \\
2 & \frac{\delta a_{\mu \nu}}{\delta r}+\xi_{; \lambda}^{\lambda} a_{\mu \nu}=0 .
\end{aligned}
$$

We rewrite Eq. (3.19c) in the following form, to which it is obviously equivalent:

$$
\xi^{2} a_{\mu \nu}-\frac{1}{\omega^{2}}\left(a_{\mu \nu: ; \lambda}^{\lambda}-R_{\varrho \mu \nu \sigma} a^{\varrho \sigma}\right)=-\frac{1}{\omega^{2}} R_{\varrho \mu \nu \sigma} a^{\varrho \sigma} .
$$

A perturbation of a given background metric described by a potential of the form (3.16) where $a_{\mu v}$ is a solution to Eqs. (3.19) in vacuo will be called a wave.

If also

$$
\left|a_{\mu \nu ; ; \lambda}^{\lambda}-R_{\varrho \mu \nu \sigma} a^{\varrho \sigma}\right| \ll \omega^{2}\left|a_{\mu \nu}\right|
$$

so that the second term on the left-hand side of Eq. (3.19e) may be put equal to zero, then we say that the wave is plane.

The reason why we have written Eq. $(3.19 \mathrm{c})$ in the form $(3.19 \mathrm{e})$ and imposed condition (3.20) as the condition that the wave be plane, will become apparent when we consider an example of a wave in a curved space-time manifold. 
In general $R_{o \mu v \sigma} a^{\varrho \sigma}$ will not be proportional to $a_{\mu v}$. This means that in general the dispersion we find will be a function of the polarization state of the wave, a phenomenon which is very important in the dispersion of electromagnetic waves if there is a background magnetic field present. We do not wish to discuss the details of this dependence here and for simplicity we shall suppose that for some function $\kappa$

$$
R_{\underline{\varrho} \mu \nu \sigma} a^{\varrho \sigma}=\kappa a_{\mu \nu} .
$$

If $a_{\mu \nu}$ is normal to a space-like vector field $p_{\mu}$ and if it is traceless, then $\kappa$ is the sectional curvature of the 2 -form normal to $p_{\mu}$ and $\xi_{\mu}$.

Eq. (3.19e) under condition (3.20) may now be rewritten as

We have set

$$
\xi^{2}=\mp \frac{\omega_{c}^{2}}{\omega^{2}} \text {. }
$$

$$
\sqrt{|\kappa|}=\omega_{c},
$$

the characteristic frequency, and the sign minus or plus is chosen according to whether $\kappa$ is positive or negative. Formula (3.22) is analogous to the classical formula for the dispersion of electromagnetic waves in a dilute plasma [3]:

$$
\xi^{2}=\omega_{p}^{2} / \omega^{2} \text {. }
$$

Before giving two examples of plane waves, we draw some immediate consequences from Eqs. (3.19), (3.20), (3.22) [4, 5]. From Eq. (3.22) we see that the hypersurfaces of constant phase are time-like or space-like according to the sign of $\kappa$. If

$$
\frac{d \omega_{c}}{d r}=0
$$

then the integral curves of the vector field $\xi^{\mu}$ are geodesics. In the limit of high frequency where the right-hand side of (3.22) may be neglected, the hypersurfaces of constant phase are null.

If we set

$$
a_{\mu v}=a n_{\mu v}, \quad n_{\mu v} n^{\mu v}=1,
$$

then from Eq. (3.19 d), we obtain the conservation equation

$$
\left(a^{2} \xi^{\mu}\right)_{; \mu}=0,
$$

and the transport equation

$$
\frac{\delta n_{\mu v}}{\delta r}=0
$$

The simplest example of a plane wave is the example in flat space. Let $\xi_{\mu}$ be an arbitrary null parallel vector field and let $a_{\mu \nu}$ be an arbitrary parallel tensor field satisfying Eq. (3.19a). Eq. (3.20) is satisfied for all $\omega$. 
An example of a gravitational wave can also be given in the non-flat manifold described in Section II. Let $a_{22}^{0}$ and $a_{23}^{0}$ be two arbitrary constants. On one of the hypersurfaces $y=$ const, introduce a tensor field whose components in the system of coordinates (2.18) are

$$
a_{\mu v}^{0}=\left[\begin{array}{cccc}
0 & 0 & 0 & 0 \\
0 & 0 & 0 & 0 \\
0 & 0 & a_{22}^{0} & a_{23}^{0} \\
0 & 0 & a_{23}^{0} & -a_{22}^{0}
\end{array}\right] .
$$

Introduce a phase given by

$$
\phi \equiv t-f(y) .
$$

Extend $a^{0}{ }_{\mu \nu}$ to a tensor field $a_{\mu \nu}$ in the region $y>0$ using Eq. (3.19d). We shall show that for $\omega$ sufficiently large $h_{\mu v}$ given by (3.16) is the potential of a plane wave. We must show that Eqs. (3.19), (3.20) are satisfied. All of the following calculations will be carried out in the coordinate system (2.18).

One easily sees that the components of the tensor field $a_{\mu v}$ will be of the form (3.26). Therefore (3.19 b) is satisfied. From (3.27) and the definition of $\xi_{\mu}$, it is obvious that (3.19a) is satisfied. Eq. (3.19d) is satisfied by construction. We are left therefore with Eqs. (3.19e) and (3.20).

A straightforward calculation yields

$$
R_{\varrho \mu \nu \sigma} a^{\varrho \sigma}=\left(\lambda^{\prime}\right)^{2} a_{\mu v} .
$$

Eq. (3.21) is therefore satisfied and the characteristic frequency $\omega_{c}$ is given by

$$
\omega_{c}=\left|\lambda^{\prime}\right| .
$$

With Eq. (3.20) satisfied, (3.22) is an equation for the function $f$ in (3.27). This equation is satisfied if we choose for $f$ any solution to the equation

$$
f^{\prime}=\sqrt{e^{-2 x}+\omega_{c}^{2} / \omega^{2}} .
$$

There remains therefore to find the conditions on $\omega$ such that condition (3.20) be satisfied.

A straightforward calculation yields the following expression for the left-hand side of the inequality (3.20):

$a_{\mu v ; ; \lambda}^{\lambda}-R_{\varrho \mu v \sigma} a^{\varrho \sigma}=-a_{\mu v}^{\prime \prime}+\left(2 \lambda^{\prime}-v^{\prime}\right) a_{\mu \nu}^{\prime}+\left(2 \lambda^{\prime \prime}+2 \lambda^{\prime} v^{\prime}+\left(\lambda^{\prime}\right)^{2}\right) a_{\mu v}$.

Using the field Eq. (2.20), this becomes

$$
a_{\mu v ; ; \lambda}^{\lambda}-R_{\varrho \mu v \sigma} a^{\varrho \sigma}=-a_{\mu v}^{\prime \prime}+\frac{5 \lambda^{\prime}}{2} a_{\mu v}^{\prime}-3\left(\lambda^{\prime}\right)^{2} a_{\mu v} .
$$


One readily sees that Eq. (3.19d) is equivalent to the following equation:

$$
a_{\mu v}^{\prime}-\lambda^{\prime} a_{\mu v}+\frac{1}{2}\left(\frac{f^{\prime \prime}}{f^{\prime}}+v^{\prime}\right) a_{\mu v}=0
$$

Suppose the following conditions are satisfied:

$$
\frac{d \omega_{c}}{d r} \ll \omega^{2}, \quad \omega_{c}^{2} \ll \omega^{2} .
$$

Then if the wave is plane, that is, if (3.20) is satisfied, by (3.22) the norm of $\xi_{\mu}$ differs very little from zero. To a first approximation we may then suppose that it is equal to zero is the Eq. (3.33) for $a_{\mu v}$. But if this is the case then the last term on the right-hand side of (3.33) vanishes. If we put the solution to the resulting equation in the right-hand side of (3.32) we find that it also vanishes. Therefore (3.20) is satisfied. We see then that a wave is plane if the frequency satisfies the inequalities (3.34).

We can in this example draw no conclusions concerning the signal velocity of a wave pulse which is a superposition of the type of plane waves considered. The phase velocity $v_{p}$ relative to a stationary observer $u^{\alpha}=\left(\sqrt{g^{00}}, 0,0,0\right)$ is given by

$$
\frac{1}{v_{p}^{2}}=1-\frac{\xi^{2}}{(u \cdot \xi)^{2}}=1+\left(\lambda^{\prime}\right)^{2} e^{2 v}>1 .
$$

Therefore $v_{p}<1$. When the dispersion relation is of the form (3.22), the group velocity is the inverse of the phase velocity. In this case it is therefore greater than one and cannot be used as the signal velocity.

The author wishes to thank Prof. A. Papapetrou for his helpful comments.

\section{References}

1. Laue, M. von: Physik. Z. XXI, 659 (1920).

2. Ehlers, J.: Z. Naturwiss. 22, 1328 (1967).

3. Stratton, J.: Electromagnetic theory. New York: McGraw-Hill 1941.

4. Isaacson, R. A.: Phys. Rev. 166, 1263, 1272 (1968).

5. Choquet-Bruhat, Y.: Commun. math. Phys. 2, 16 (1969); -- C. R. Acad. Sc. 258, 1089 (1964).

6. Kasner, E.: Trans. Am. Math. Soc. 27, 155 (1925).

\section{J. Madore}

Physique Théorique

Institut Henri Poincaré

11 Rue Pierre et Marie Curie

F-75 Paris V, France 